\title{
Electronic Nose Coupled with Linear and Nonlinear Supervised Learning Methods for Rapid Discriminating Quality Grades of Superior Java Cocoa Beans
}

\author{
Shidiq Nur Hidayat ${ }^{1}$ \\ Aldin Rusman ${ }^{1}$ \\ Trisna Julian ${ }^{1}$ \\ Kuwat Triyana ${ }^{1,2 *}$ \\ Ana C.A. Veloso ${ }^{3,4}$ \\ António M. Peres ${ }^{5,6}$ \\ ${ }^{I}$ Physics Department, Universitas Gadjah Mada, Sekip Utara BLS 21, Yogyakarta, 55281 Indonesia \\ ${ }^{2}$ Institute of Halal Industry and System (IHIS), \\ Universitas Gadjah Mada, Sekip Utara, Yogyakarta, 55281 Indonesia \\ ${ }^{3}$ Instituto Politécnico de Coimbra, ISEC, DEQB, Rua Pedro Nunes, Quinta da Nora, 3030-199 Coimbra, Portugal \\ ${ }^{4}$ CEB - Centre of Biological Engineering, University of Minho, Campus de Gualtar, 4710-057 Braga, Portugal \\ ${ }^{5}$ Centro de Investigação de Montanha (CIMO), ESA, \\ Instituto Politécnico de Bragança, Campus Sta Apolónia, 5300-253 Bragança, Portugal \\ ${ }^{6}$ Laboratory of Separation and Reaction Engineering - Laboratory of Catalysis and Materials (LSRE-LCM), ESA, \\ Instituto Politécnico de Bragança, Campus Santa Apolónia, 5300-253 Bragança, Portugal \\ * Corresponding author's Email: triyana@ugm.ac.id
}

\begin{abstract}
An electronic nose (E-nose), comprising eight metal oxide semiconductor (MOS) gas sensors and a moisture-temperature sensor, was used for classifying three quality grades of superior java cocoa beans, namely fine cocoa dark bean $<20 \%$, fine cocoa dark bean $>60 \%$, and bulk cocoa bean that is a harder task compared to the discrimination of high versus low-quality cocoa beans. The E-nose signals were pre-processed using the maximum value method. The capability for discriminating the quality grade of the cocoa beans was checked by applying multivariate statistical tools, namely, linear discriminant analysis (LDA), support vector machine (SVM) and artificial neural networks (ANN). For this, the experimental dataset was split into two subsets, one for training (i.e., establishing the classification models) and the other for external-validation purposes. Furthermore, hyperparameter optimization and $\mathrm{K}$-fold cross-validation variant were implemented during the model training procedure to select the best classification models and to avoid over-fitting issues. The best predictive classification performance was obtained with the E-nose-MLP-ANN procedure, which allowed 99\% of correct classifications (overall accuracy) for the training dataset and 95\% of correct classifications (overall accuracy) for the external-validation dataset. The satisfactory results clearly demonstrated that the E-nose could be applied as a quality control tool in the cocoa industry, requiring minimum and simple sample preparation.
\end{abstract}

Keywords: Cocoa bean quality, Electronic nose, Linear discriminant analysis, Artificial neural networks, Support vector machines.

\section{Introduction}

Cocoa (Theobroma cacao L.) is an important agricultural commodity in the world with a global annual market value of US $\$ 5.1$ billion [1]. The World Cocoa Foundation estimates that, worldwide, 40-50 million people economically depend on cocoa [1-3]. Cocoa has a unique aroma and taste, being a popular product, especially after processed into chocolate [4]. Increased demand for high-quality cocoa products has arisen worldwide, which production can only be ensured by using highquality cocoa beans. In 2016, 4,400,000 tons of cocoa beans were produced, worth US $\$ 98.3$ billion [5]. Therefore, quality control is required to ensure the quality of the cocoa beans. In this sense, different non-destructive conventional analytical techniques have been reported in the literature for 
assessing cocoa geographical origin, variety or grade quality. For example, cocoa bean's fermentation index has been predicted based on hyperspectral imaging [4] or based on colour analysis [6]. Near-Infrared (NIR) spectroscopy or Fourier Transform Near-Infrared (FT-NIR) spectroscopy, coupled with multivariate statistical tools, have been used as non-destructive techniques, to assess cocoa bean geographical origin or quality grades [2, 7-9]. A NIR spectroscopy-electronic tongue integrated approach was accurately used for classifying cocoa bean varieties $[10,11]$. Other researchers showed that Mass Spectrometry (MS)fingerprinting could be a reliable and fast method for predicting the aroma potential and quality of roasted cocoa beans, the extent of processing as well as their geographical origin [12]. The influence of the roasting process conditions on the polyphenol contents of cocoa beans, nibs and chocolates have been evaluated by UPLC-DAD-ESI-MS/MS [13]. On the other hand, chocolate polyphenol profiles, established by high-performance liquid chromatography-diode array detector-mass spectrometry analysis (HPLC-DAD-MS), allowed the successful discrimination of cocoa beans by geographical origin and variety [14]. Also, sensory analysis together with liquid chromatography (LC) have been described to assess the $\mathrm{pH}$-dependency of the proteolytic formation of cocoa- and nuttyspecific aroma precursors [15] as well as to characterize the peptide precursors of the cocoaspecific aroma components [16]. More recently, an LC Time-of-Flight Mass Spectrometry (LC-TOFMS)-based metabolomics approach was developed for assessing the geographical origin of cocoa beans, using partial least squares discriminant analysis [17]. Multi-elemental fingerprinting also enabled the geographic traceability of Theobroma cacao beans and cocoa products [18]. A classification chemometric model based on HR-MS 1H NMR data has been also proposed for assessing the geographical origin of cocoa beans [19]. Finally, cocoa fermentation quality and aroma formation potential have been evaluated by gas chromatography-mass spectrometry (GC-MS) [12], [20]. Indeed, advanced chemical untargeted and targeted fingerprinting together with twodimensional GC-MS may be used as a pattern recognition tool for classification, discrimination, and sensory-quality assessment of high-quality cocoa at early processing stages [21]. In fact, the volatile flavour profile is one of the most important factors in determining the quality of cocoa beans. Cocoa beans have a unique complex volatile profile that is responsible for its unique and highly appreciated aroma, which is the result of a mixture of 600 different chemical compounds, such as alcohols, aldehydes, ketones, acids, esters, and pyrazines [22].

For cocoa analysis, NIR spectroscopy provides rapid analysis for the conventional method, but it is relatively high-cost equipment and needs experts to operate. On the other hand, HPLC, LC-MS, GC-MS, and NMR provide precise and reliable information about compounds of a sample, but they are expensive and need specific sample preparation, i.e., GC-MS need sample extraction and preconcentration to get precise results that need expert [23]. Therefore, the above mentioned conventional analytical techniques being usually far beyond the economic capacity of several local cocoa industries.

In this context, electronic noses (E-nose) have emerged as possible alternative analytical tools for cocoa evaluation, based on their sensitivity towards volatile organic compounds (VOC) that are responsible for the specific and exclusive cocoa aroma. Indeed, E-nose is usually low-cost devices, user-friendly interfaces and do not require complex sample pre-treatments nor experienced operators. The E-nose is a device that aims to mimic the working concept of the human nose through a set of gas sensor arrays that are able to detect and distinguish different VOCs [24-27]. So, some studies reported the use of E-noses for cocoa bean analysis. The quality of cocoa beans could be accurately assessed using different E-nose devices coupled with chemometric tools, such as extreme machine learning (ELM), support vector machine (SVM), linear discriminant analysis (LDA), knearest neighbours (k-NN) and artificial neural networks (ANN), with accuracy rates of $89 \%$ to $95 \%[9,28]$. On the other hand, cocoa beans degree of roasting could also be assessed coupling an Enose (MOS sensors) with ANN model, with a predictive accuracy of $94.4 \%$, similar to that of a GC-MS (95.8\%) [5]. Likewise, an E-nose, with six MOS gas sensors, was used to continuously monitor VOC of cocoa samples undergoing refining, using a Kernel distribution model, allowing predicting optimal refining and conching times [29]. Finally, it has been demonstrated that a GC E-nose could be an effective and fast aroma profiling allowing discrimination between single-origin cocoa liquors [22].

In the present study, we reported the overall satisfactory performances of cocoa analysis with an E-nose device. The three cocoa bean quality grades used in this study are superior quality and so this is a harder task compared to the discrimination of high 
Table. 1 Cocoa bean samples details

\begin{tabular}{ccccl}
\hline Label & $\begin{array}{c}\text { High-grade Java } \\
\text { Cocoa Type }\end{array}$ & $\begin{array}{c}\text { Number } \\
\text { of } \\
\text { samples }\end{array}$ & $\begin{array}{c}\text { Sample code } \\
\text { from factory }\end{array}$ & Additional information \\
\hline A & $\begin{array}{l}\text { Fine cocoa: dark } \\
\text { bean <20\% }\end{array}$ & 100 & I-AA-FC/W & $\begin{array}{l}\text { Criollo or Trinitario cocoa varieties } \\
\text { subjected to a short fermentation } \\
\text { time }\end{array}$ \\
B $\quad \begin{array}{l}\text { Fine cocoa: dark } \\
\text { bean > 60\% }\end{array}$ & 100 & I-AA-FC/W & $\begin{array}{l}\text { Criollo or Trinitario cocoa varieties } \\
\text { subjected to a long fermentation } \\
\text { time }\end{array}$ \\
C $\quad$ Bulk cocoa & 100 & I-B-BC/W & Forastero variety \\
\hline
\end{tabular}

${ }^{a}$ Quality grades according to the Indonesian National Standard (SNI 2323:2008) [30]

versus low-quality cocoa beans. Thus, in this work, we demonstrated the capability of a lab-made Enose, with eight different MOS gas sensors and a combined moisture-temperature sensor, was built and applied for differentiating three grades of superior java cocoa bean samples, using linear (LDA) and nonlinear (SVM and ANN) supervised pattern recognition statistical tools. This paper is organized into the following sections: Section 1 and 2 explain the background and materials and methods related to the work, respectively. Meanwhile, Section 3 describes the results and discussion. Finally, section 4 explains the conclusion of this work.

\section{Materials and methods}

\subsection{Sample preparation}

Java cocoa bean samples, with different quality grades, were acquired from the plantation factory PTPN XII (Surabaya, Indonesia). Two types of cocoa beans were obtained, namely fine cocoa and bulk cocoa, which classifications are in accordance with the Indonesian National Standard (SNI 2323:2008) [30]. In this study, bulk cocoa and two kinds of fine cocoa (i.e., fine dark cocoa bean $>60 \%$ and fine dark cocoa bean $<20 \%$ ) were studied. Table 1 shows these three cocoa bean quality grades that are superior quality or high-grade. The cocoa beans were previously fermented at the factory. For replication purposes, sub-samples from each cocoa quality code were analyzed (100 independent assays) allowing to establish a dataset with 300 data for each type of cocoa (100 assays $\times 3$ types of cocoa beans).

\subsection{Electronic nose apparatus and analysis}

A lab-made E-nose with eight MOS gas sensors (TGS series) along with relative humidity and temperature sensor, was used to analyze cocoa bean samples. The eight TGS series of gas sensor (TGS 822, TGS 2612, TGS 2620, TGS 832, TGS 826, TGS 2603, TGS 2600, TGS 813) were equipped in the device as well as the most targeted gases as listed in our previous work [27]. Here, the Sensirion SHT-31 sensor was used as relative humidity and temperature sensor. Fig. 1 the measurement system of E-nose used in this study. Briefly, the E-nose consisted of a sampling system, a sensor array system, a data acquisition system (DAQ), and a signal processing framework as illustrated in Fig. 2. Two valves (valve-1 and valve-2) were used in the sampling system to control the air flow passing through the E-nose system. The air flow reached the sensor chamber after a two-step procedure of delay/purging step and sampling step. During the delay/purging step, air (from air reference or air ambient) flows directly into the sensor chamber. Oppositely, during the sampling step, the air is directed through the sample container and then enters the sensor chamber. In this step, volatile organic compounds (VOC) from the sample are carried by the air flow and allowed to enter the sample container for detection. The DAQ used a 16bit ADC and an Arduino Mega microcontroller. Prior to measuring, a 2-bean of the cocoa sample 


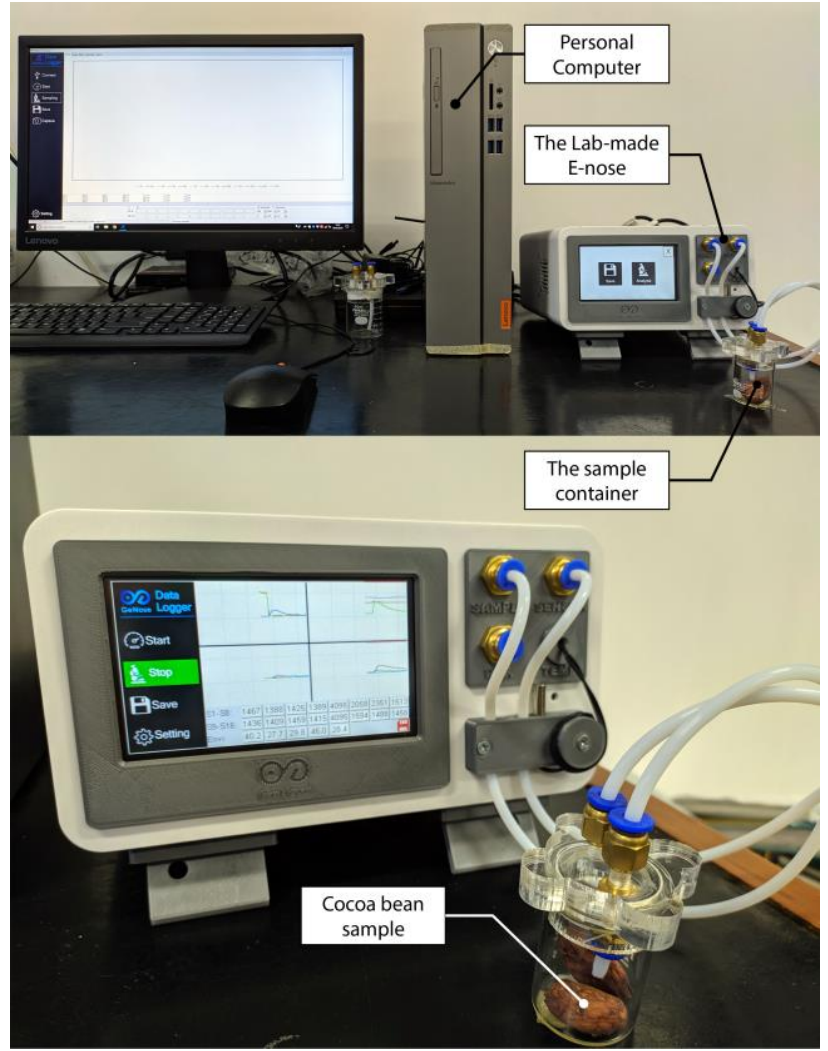

Figure. 1 The measurement system developed

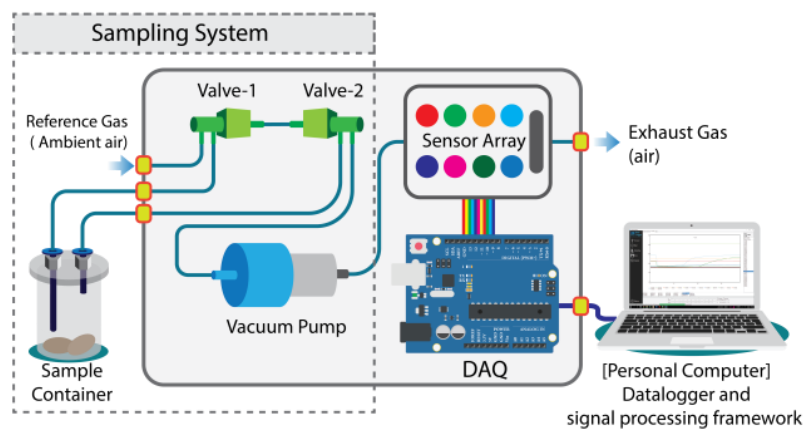

Figure. 2 Schematic diagram of the E-nose device

was put into a $10-\mathrm{mL}$ beaker glass (sample container, Fig. 1) and analyzed with the E-nose, being settled a time delay phase of $60 \mathrm{~s}$, a sampling phase of $60 \mathrm{~s}$, and a purging phase of $180 \mathrm{~s}$. The measurements were carried out at room temperature $\left(25^{\circ} \mathrm{C}\right)$ and without heating the sample.

\subsection{Data pre-processing}

The signals data matrix generated by the E-nose consisted of 10 signal profiles gathered by the 8 MOS gas sensors and the moisture-temperature sensor. For each sample assay the data matrix contained 10 sensors $\times 3,000$-raw time series values. One of the signals recorded is shown in Fig. 3 that was recorded using the lab-made E-nose and DAQ system for cocoa fine dark $<20 \%(\operatorname{cocoa} A)$. Each $i$ -

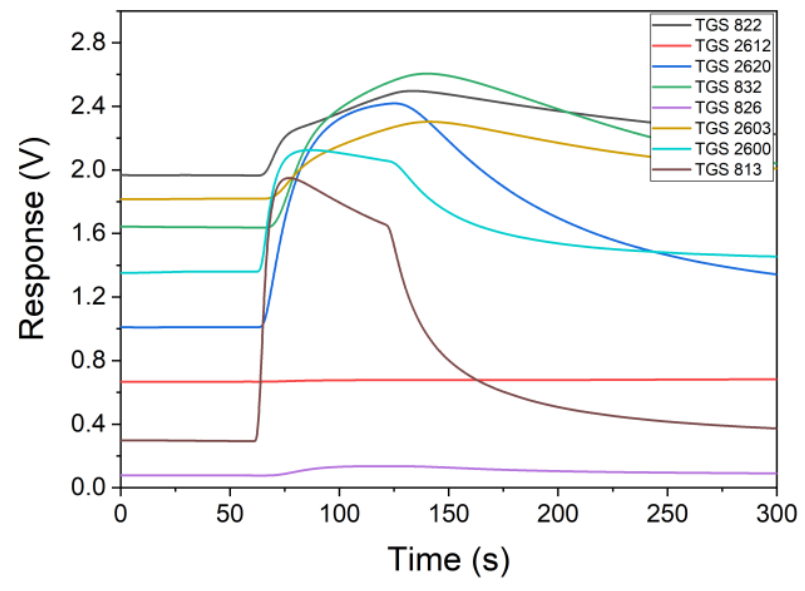

Figure. 3 One of the signals recorded using the lab-made E-nose for the fine dark cocoa bean $<20 \%$ samples ( cocoa A)

sensor and $j$-sample produces a signal with a time series $(t), V_{i, j}(t)$. For the statistical analysis, the raw data matrix was pre-processed using a feature extraction method. The feature extraction method aims to retrieve important information $\left(X_{i, j}\right)$ of the profile of the signal and minimize the redundant data [31]. In this study, the statistical parameter maximum signal value of the raw E-nose data was used as a feature extraction method, which can be represented by Eq. (1). Before analysis, the preprocessed data was normalized using scaled (Eq. (2)) and centered (Eq. (3)) methods, where $x_{i}$ is the data point $i, x^{\prime}{ }_{i}$ is the transformation data point $i, \bar{x}_{l}$ is the mean value of $x_{i}$ and $\delta x_{i}$ is the standard deviation of $x_{i}$.

$$
\begin{aligned}
& X_{i, j}=\max \left(V_{i, j}(t)\right) \\
& x^{\prime}{ }_{i}=\bar{x}_{l}-x_{i} \\
& x^{\prime}{ }_{i}=\frac{x_{i}}{\delta x_{i}}
\end{aligned}
$$

\subsection{Proposed methods}

After data pre-processing, the final data matrix comprising 10 sensors $\times 300$ maximum value was built, for all cocoa samples evaluated. This preprocessed dataset was split into two data subsets using the Kennard-Stone algorithm [32], one containing $80 \%$ of the data that was used for training (i.e., for establishing the classification chemometric models) and the other, with the remaining $20 \%$ of the data, for testing (i.e., for external-validation of the developed models). For the statistical analysis, cocoa samples were grouped 


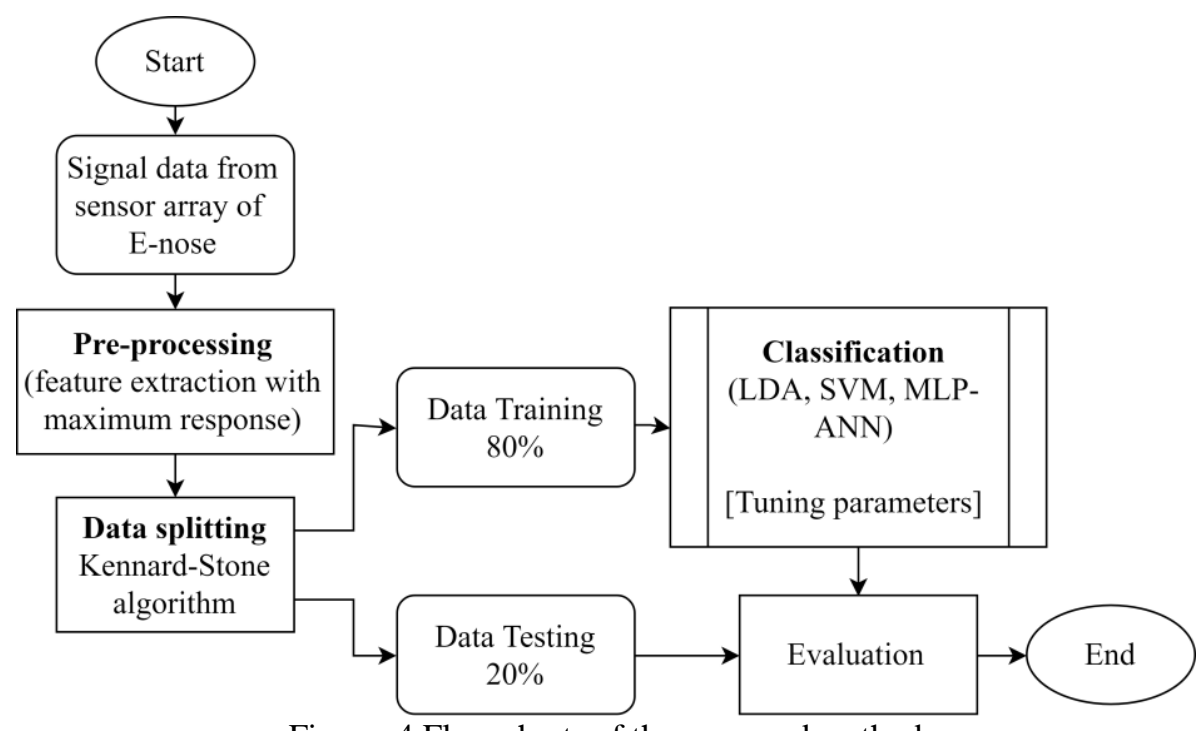

Figure. 4 Flow charts of the proposed method

according to the quality grade using the class labels $\mathrm{A}, \mathrm{B}$, and $\mathrm{C}$ as listed in Table 1. Fig. 4 shows the flow chart of the proposed method to determine the performance of the E-nose to classify the samples.

First, linear discriminant analysis (LDA), a supervised multivariate statistical method, was applied for evaluating the possibility of establishing a linear discriminant model, based on the signal profiles gathered by the E-nose sensors, for classifying the cocoa beans by quality grade. Then, non-linear multivariate models were also evaluated, considering the expected non-linear characteristics of the MOS gas sensors signals. Support vector machine (SVM) and artificial neural networks (ANN) were used aiming to verify the feasibility of classifying the cocoa bean samples according to the pre-known quality grades. SVM is a machine learning technique that is able to handle data with linear and non-linear characteristics, allowing to deal with complex pattern recognition problems [33]. SVM is a classifier technique that uses a quadratic hyperplane optimization to discriminate the classes under study. The kernel function generally used to optimize the performance of SVM is a liner, polynomial or a radial basis kernel function. SVM has hyperparameters like the cost value (C) and the gamma value. In this work, a grid search method (exhaustive search method) was implemented to select the optimal hyperparameters in order to establish the best SVM classification model. K-fold cross-validation was implemented in the grid search process to overcome over-fitting issues.

Furthermore, ANN was also used. In this case, a multilayer perceptron (MLP) architecture, with a feed-forward algorithm, was applied for cocoa bean samples classification by quality grade. MLP is a nonlinear model that requires setting or optimizing several hyperparameters, namely, the number of neurons, the number of hidden layers, the activation functions, the dropout layer, the loss function, and the optimizer [34]. In theory, the number of hidden layers and the number of neurons is unlimited.

The rectified linear unit (ReLU) function, the sigmoid function, the softmax function, and leaky ReLU function are commonly used as the activation function. The dropout layer is usually included to overcome possible over-fitting problems. The loss function is a metric that helps the model to understand whether the learning procedure is in the right direction or not, after each iteration. Since this study involved a multiclass classification task, the selected loss function was a categorical crossentropy method. On the other hand, the backpropagation algorithm was selected as the optimization algorithm [33, 35]. In this work, two hidden layers with 300 neurons and 100 neurons and $\mathrm{ReLu}$ activation function were used for establishing the MLP-ANN model. To avoid over-fitting, the MLP-ANN model used a dropout layer with a value of 0.2 and contained 100 neurons. A softmax function was used in the output layer that contains 3 neurons to handle multiclass classification.

Fig. 5 shows the proposed architecture of the MLP-ANN-E-nose. The MLP-ANN-E-nose model used a Glorot uniform for the initial kernel, a categorical cross-entropy for the loss function, an Adam algorithm for the optimizer, and five batch size. The modelling development and analysis were performed using the Python 3.6 open-source software with scikit-learn and keras libraries [33], [34]. In this works, an accuracy metric was used to compare the performance of classification models. The accuracy can be calculated from Eq. (4), 


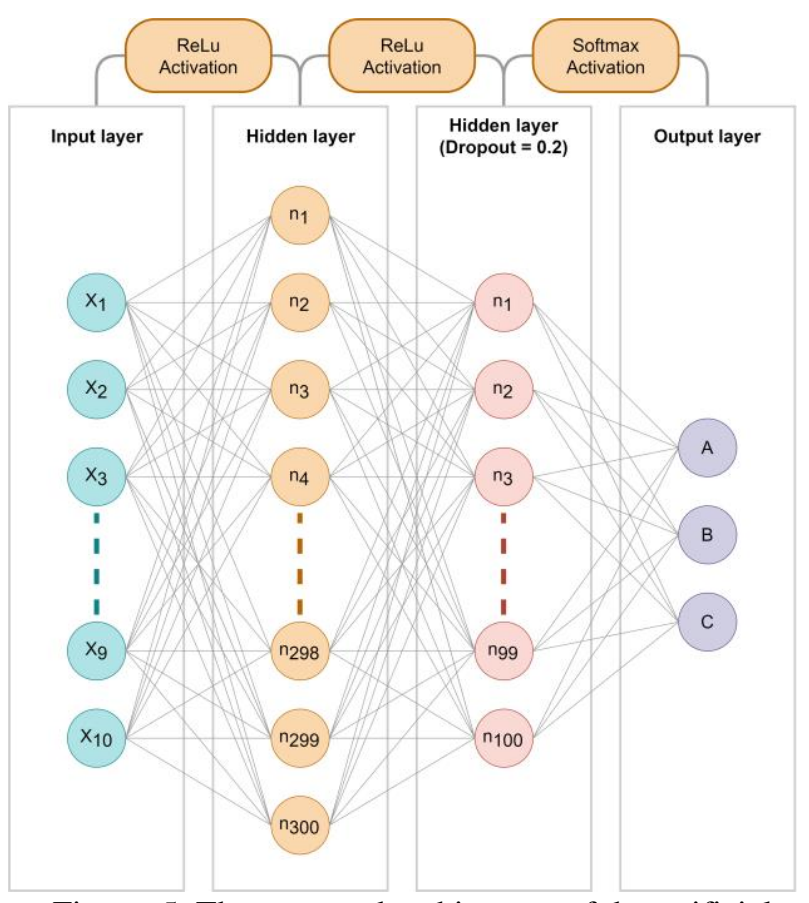

Figure. 5 The proposed architecture of the artificial neural networks of multiple layer perceptron (MLP)

$$
\% \text { Accuracy }=\frac{T P+T N}{T P+T N+F P+F N} \times 100 \%
$$

where $T P$ is true positive, $T N$ is true negative, $F P$ is false positive, and $F N$ is a false negative.

\section{Results and Discussion}

In this case of the cocoa bean analysis, no specific sample treatment is needed for measuring using the E-nose. During the training and testing processes, each assay took place a relatively short time, about five minutes, for analysis using the Enose. It included delay, sampling and purging processes. Moreover, the E-nose has been designed for very easy in operation and interpretation and for another case, it is suitable for in-situ measurement [23].

The mean signal profiles of eight MOS gas sensors (centred and scaled) obtained from the labmade E-nose for each cocoa bean sample is shown in Fig. 6, using a radar plot. It shows the variability of the signal profiles recorded by each sensor of the lab-made E-nose for the cocoa bean samples that could be attributed to the different volatile organic compound of the cocoa bean samples. To confirm this hypothesis, the discrimination analysis method (LDA) was performed using the signals data matrix in order to verify the distribution of all assays for classification purpose. Moreover, LDA was used to classify the data.

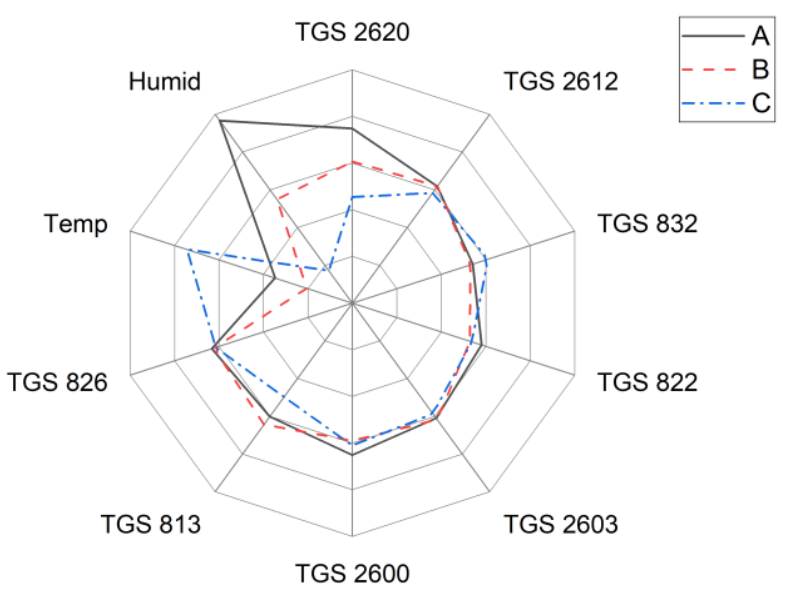

Figure. 6 Radar plots of the average responses obtained with the lab-made E-nose

The performance of the LDA was pointed out in Fig. 7 that it was possible to establish a classification model, based on the signals recorded by the E-nose during the analysis of the cocoa beans. The model comprised two discriminant functions (LD1 and LD2), which explained $83.4 \%$ and $16.6 \%$ of the total variance. The linear classification LDAE-nose model allowed to correctly identify the cocoa quality grade of $70 \%$ of the original grouped samples (for the training dataset) and of $55 \%$ for the cocoa samples included in the testing dataset (i.e., external validation). The established model showed and under-fitting performance regarding the prediction of the quality of cocoa beans. As can be inferred from Fig. 7, samples belonging to the three cocoa quality grades overlap, which is more evident between samples belonging to the fine dark cocoa bean $>60 \%$ (B) and the bulk cocoa bean (C) that have individual class sensitivities of $70 \%$ (B), $68 \%$ (C) for data training and $0 \%(B), 28 \%(C)$ for data testing. Furthermore, the model showed specificities of $80 \%$ (B), $84 \%$ (C) for data training and $82.4 \%$ (B), $76.9 \%$ for data testing. The overall results showed that the LDA-E-nose was not capable of discriminating cocoa quality $\mathrm{B}$ and $\mathrm{C}$ with the required sensitivity, which would be an important drawback for practical applications. On the contrary, samples of fine dark cocoa bean $<20 \%$ (A) were the most easily discriminated with a sensitivity of $77 \%$ for data training and $90 \%$ for data testing; and with a specificity of $90 \%$ for data training and of $70 \%$ for data testing. The less satisfactory performance of the LDA-E-nose model pointed out the need for applying other supervised pattern recognition models, which could deal with linear and non-linear data behaviours. 


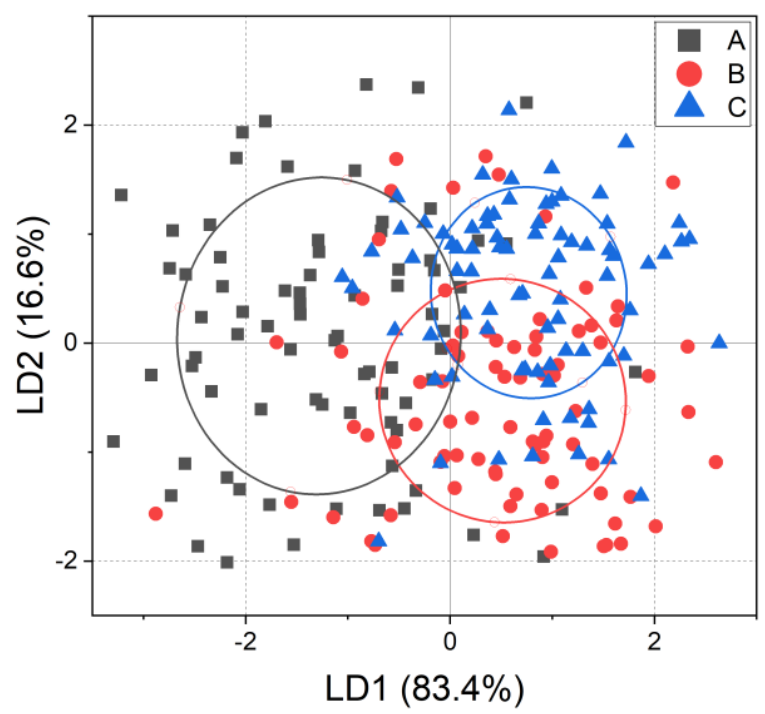

Figure. 7 LDA-E-nose classification performance of cocoa bean samples according to the quality grades (A) fine dark cocoa bean <20\%; (B) fine dark cocoa bean > $60 \%$; and, (C) bulk cocoa bean

So, an SVM-E-nose model was developed using the grid search method to determine the best model hyperparameters. In addition, to minimize the risk of over-fitting, a 10-fold cross-validation procedure (i.e., an internal cross-validation variant) was used within the grid search procedure. The 10 -fold crossvalidation implies a random split of the training dataset into 10 subsets, each one comprising $10 \%$ of training data, being, at each run, one subset used for internal-validation of the SVM model established using the other nine data subsets. Moreover, for the grid search procedure, a linear kernel and an RBF kernel functions were used. The $\mathrm{C}$ was set to 0.01 , $0.1,1.0,10.0,100.0$, and 1000.0 while the gamma was set to $0.001,0.01,0.1,1.0,10.0,100.0$, and 1000.0. The applied procedure allowed selecting using the RBF kernel function, a $\mathrm{C}$ value of 100.0 and a gamma value of 0.1 as the values of the optimal hyperparameters of the best SVM-E-nose classification model. This SVM-E-nose model had an overall predictive accuracy of $99 \%$ for the original grouped data (training data) and an overall predictive accuracy of $90 \%$ for the testing data (sensitivity of $90 \%, 100 \%$ and $87 \%$ for cocoa quality grades $\mathrm{A}, \mathrm{B}$, and $\mathrm{C}$, respectively).

Although the classification performance of the SVM-E-nose model was satisfactory, an MLP-ANN technique was further applied. The MLP-ANN-Enose model was developed using a sequential procedure. The ANN model enabled the correct assessment of the quality grade of all cocoa bean samples for the training dataset (an overall

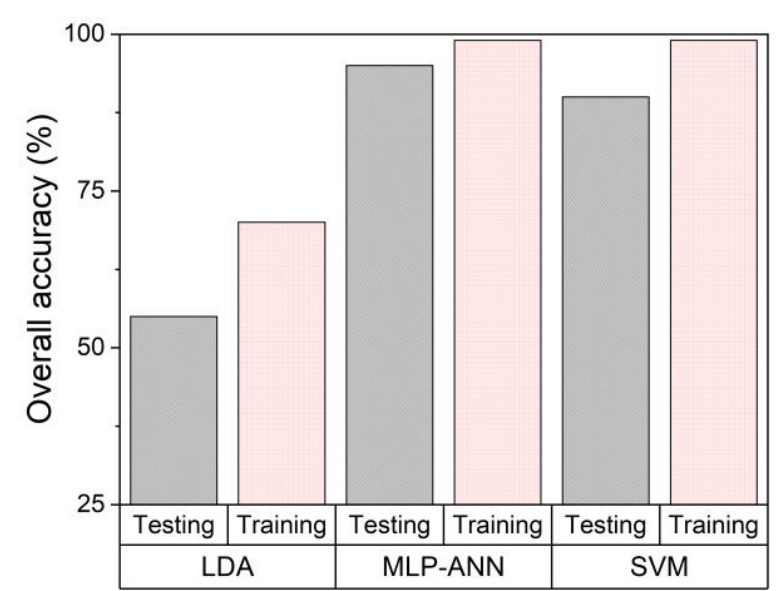

Figure. 8 Accuracy achieved for training (black bars), and external-validation (testing dataset, red bars) for the combinations of supervised pattern recognition tool (LDA, MLP-ANN, and SVM)

Table. 2 The overall sensitivities and specificities of the developed supervised E-nose classification models

\begin{tabular}{cccccccc}
\hline & & \multicolumn{3}{c}{ Sensitivity (\%) } & \multicolumn{3}{c}{ Specificity $(\%)$} \\
\cline { 3 - 8 } & & $\mathbf{A}$ & $\mathbf{B}$ & $\mathbf{C}$ & $\mathbf{A}$ & $\mathbf{B}$ & $\mathbf{C}$ \\
\hline \multirow{2}{*}{ LDA } & Train & 77 & 70 & 68 & 90 & 80 & 84 \\
& Test & 90 & 0 & 28 & 70 & 82 & 77 \\
\hline \multirow{2}{*}{ SVM } & Train & 100 & 100 & 97 & 100 & 98 & 100 \\
& Test & 90 & 100 & 86 & 100 & 88 & 100 \\
\hline \multirow{2}{*}{ MLP } & Train & 99 & 99 & 100 & 100 & 100 & 100 \\
- & Test & 100 & 100 & 86 & 100 & 94 & 100 \\
ANN & Ten
\end{tabular}

predictive accuracy equal to $99 \%$ ) and a global predictive classification accuracy of $95 \%$ for cocoa samples of the testing dataset (sensitivities of 100\%, $100 \%$ and $86 \%$ for $\mathrm{A}, \mathrm{B}$ and $\mathrm{C}$ quality grades, respectively).

Fig. 8 shows the overall accuracies and Table 2 shows a comparison between the overall sensitivities and specificity obtained with the different E-nose classification models developed for the cocoa quality grade discrimination, based on LDA, SVM, and MLP-ANN approaches.

The results showed that both SVM and MLPANN could be successfully used for assessing the cocoa beans quality grade when 3 classes were evaluated, namely high-grade java fine dark cocoa bean $<20 \%$ (A), high-grade java fine dark cocoa bean $>60 \%$ (B), and high-grade java bulk cocoa bean $(\mathrm{C})$.

Fig. 9 shows the confusion matrix of the MLPANN from the classification of the overall samples. Generally, a confusion matrix performed the performance of a classification method. In this work, 4 data were predicted incorrectly by the MLP-ANN. 


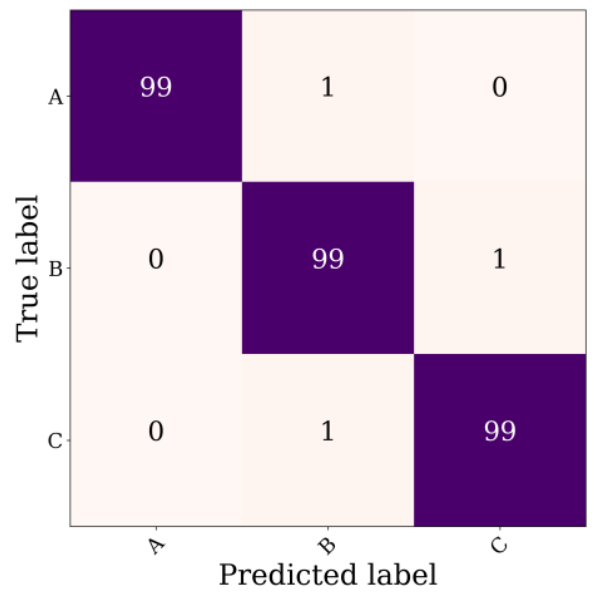

Figure. 9 Result of confusion matrix MLP-ANN for classification all the superior Java cocoa bean samples

Table. 3 Performance measurement of MLP-ANN for classification all the superior Java cocoa bean samples

\begin{tabular}{lccc}
\hline & A & B & C \\
\hline PPV & 1.00 & 0.98 & 0.99 \\
TNR & 1.00 & 0.99 & 0.99 \\
TPR & 0.99 & 0.99 & 0.99 \\
F1-Score & 0.99 & 0.98 & 0.99 \\
\hline Overall Accuracy & & 0.99 & \\
Kappa & & 0.98 & \\
\hline
\end{tabular}

From the confusion matrix, we can calculate other performance indicators, such as accuracy, kappa, precision or positive prediction value (PPV), true negative rate (TNR) or specificity, true positive rate (TPR) or sensitivity/recall/hit rate, and F1-Score. Table 3 shows the value of these indicators for the MLP-ANN from classifying overall samples. Generally, we can get the best classification performance of discrimination each of three quality superior Java cocoa bean samples.

Furthermore, the performances achieved in the present study are in agreement with those reported by Olunloyo et al. that used an E-nose coupled with ANN to assess the quality grade of cocoa beans (overall accuracy of 95\%) [28]. However, it should be remarked that in this later work, the cocoa beans belonged to very different quality grades, namely high and low-quality grades. On the contrary, in the present study, it was aimed to distinguish 3 different types of high-quality cocoa beans. Slightly better classification performances were obtained compared to those reported by Kutsanedzie et al., for the classification of cocoa beans from 3 quality grades (fully fermented, partially fermented and nonfermented) using an E-nose coupled with LDA, SVM or k-NN models (accuracies from 89 to $94 \%$ ) [9]. The overall results reported in the present study allowed verifying that the E-nose, comprising MOS gas sensors and a moisture-temperature sensor, coupled with suitable supervised classification models (SVM or MLP-ANN) can be used as a practical device to correctly discriminating cocoa bean samples according to their quality grades, being possible to infer that fine dark cocoa bean < $20 \%$ and fine dark cocoa bean $>60 \%$ possessed different aroma fingerprints even though they belong to the same cocoa class, i.e., fine cocoa beans. Contrary, bulk cocoa beans and fine dark cocoa beans $>60 \%$, apparently have more similar aroma attributes. Since, bulk cocoa beans have a strong aroma and flavor characteristics, it can be tentatively concluded that the fine dark cocoa bean > $60 \%$ has a stronger aroma characteristic than the fine dark cocoa bean $<20 \%$. A fine cocoa bean is usually more aromatic and so, has a smoother aroma. The taste and aroma consistency of the cocoa beans is related to the flavor, acidity, sourness, bitterness, and astringency, which intensities are influenced by cocoa bean variety, geographical origin and agricultural practices $[2-7,12,22,35]$. Generally, cocoa beans become darker over a long period of fermentation, due to the presence of anthocyanin pigments, being the final flavor of cocoa highly dependent on the fermentation level $[2,4]$.

\section{Conclusions}

This study demonstrated the prospective of using a lab-made E-nose, with MOS gas sensors, coupled with chemometric tools to correctly classify the quality grade of java cocoa beans (fine dark cocoa bean $<20 \%$, fine dark cocoa bean $>60 \%$, and bulk cocoa bean). The best predictive classification performance was obtained with the MLP-ANN procedure, which allowed 99\% overall accuracy of correct classifications for all datasets with high sensitivity and specificity. The overall satisfactory predictive performance achieved with the E-nose device pointed out the possibility of its practical implementation at an industrial level, which may be foreseen in the future. Indeed, the proposed tool allowed a preliminary, fast and cost-effective assessment of the cocoa bean quality, being an alternative or at least a complementary technique of common conventional high-cost and timeconsuming analytical techniques.

\section{Acknowledgments}

The authors thank the Directorate of Research and Community Service, Ministry of Research, Technology and Higher Education, the Republic of Indonesia for providing research grants of PTUPT 2019 (Contract No. 2688/UN1.DITLIT/DITLIT/LT/2019). The authors also like to acknowledge 
the financial support given by Associate Laboratory LSRE-LCM-UID/EQU/50020/2019, strategic funding UID/BIO/04469/2019-CEB, BioTecNorte operation (NORTE-01-0145-FEDER-000004) and strategic project PEst-OE/AGR/UI0690/2014 CIMO, all funded by national funds through FCT/MCTES (PIDDAC).

\section{References}

[1] E. Ohene, Cocoa Production Processing Technology. New York: CRC Press, 2014.

[2] S. Sunoj, C. Igathinathane, and R. Visvanathan, "Nondestructive determination of cocoa bean quality using FT-NIR spectroscopy", Comput. Electron. Agric., Vol. 124, pp. 234-242, 2016.

[3] F. Gu, L. Tan, H. Wu, Y. Fang, F. Xu, Z. Chu, and Q. Wang, "Comparison of Cocoa Beans from China, Indonesia and Papua New Guinea", Foods, Vol. 2, No. 2, pp. 183-197, 2013.

[4] N. Caporaso, M. B. Whitworth, M. S. Fowler, and I. D. Fisk, "Hyperspectral imaging for nondestructive prediction of fermentation index, polyphenol content and antioxidant activity in single cocoa beans", Food Chem., Vol. 258, pp. 343-351, 2018.

[5] J. Tan and W. L. Kerr, "Determining degree of roasting in cocoa beans by artificial neural network (ANN)-based electronic nose system and gas chromatography/mass spectrometry (GC/MS)," J. Sci. Food Agric., Vol. 98, No. 10, pp. 3851-3859, 2018.

[6] N. León-roque, M. Abderrahim, L. Nuñezalejos, S. M. Arribas, and L. Condezo-hoyos, "Prediction of fermentation index of cocoa beans ( Theobroma cacao L .) based on color measurement and arti fi cial neural networks", Talanta, Vol. 161, pp. 31-39, 2016.

[7] E. Teye, X. Huang, L. K. Sam-Amoah, J. Takrama, D. Boison, F. Botchway, and F. Kumi, "Estimating cocoa bean parameters by FTNIRS and chemometrics analysis", Food Chem., Vol. 176, pp. 403-410, 2015.

[8] [8] E. Teye, X. Huang, H. Dai, and Q. Chen, "Rapid differentiation of Ghana cocoa beans by FT-NIR spectroscopy coupled with multivariate classification", Spectrochim. Acta - Part A Mol. Biomol. Spectrosc., Vol. 114, pp. 183-189, 2013.

[9] F. Y. H. Kutsanedzie, Q. Chen, H. Sun, and W. Cheng, "In situ cocoa beans quality grading by near-infrared-chemodyes systems", Anal. Methods, Vol. 9, No. 37, pp. 5455-5463, 2017.

[10] E. Teye, X. Huang, J. Takrama, and G. Haiyang, "Integrating NIR spectroscopy and electronic tongue together with chemometric analysis for accurate classification of cocoa bean varieties", J. Food Process Eng., Vol. 37, No. 6, pp. 560566, 2014.

[11] E. Teye, X. Huang, F. Han, and F. Botchway, "Discrimination of Cocoa Beans According to Geographical Origin by Electronic Tongue and Multivariate Algorithms", Food Anal. Methods, Vol. 7, No. 2, pp. 360-365, 2014.

[12] P. D. Tran, D. Van de Walle, N. De Clercq, A. De Winne, D. Kadow, R. Lieberei, K. Messens, D. N. Tran, K. Dewettinck, and J. Van Durme, "Assessing cocoa aroma quality by multiple analytical approaches", Food Res. Int., Vol. 77, pp. 657-669, 2015.

[13] D. Żyżelewicz, W. Krysiak, J. Oracz, D. Sosnowska, G. Budryn, and E. Nebesny, "The influence of the roasting process conditions on the polyphenol content in cocoa beans, nibs and chocolates", Food Res. Int., Vol. 89, pp. 918929, 2016.

[14] A. Cambrai, E. Marchioni, D. Julien-David, and C. Marcic, "Discrimination of Cocoa Bean Origin by Chocolate Polyphenol Chromatographic Analysis and Chemometrics", Food Anal. Methods, Vol. 10, No. 6, pp. 19912000, 2017.

[15] J. Voigt, K. Textoris-Taube, and J. Wöstemeyer, "pH-Dependency of the proteolytic formation of cocoa- and nutty-specific aroma precursors", Food Chem., Vol. 255, pp. 209-215, 2018.

[16] J. Voigt, K. Janek, K. Textoris-Taube, A. Niewienda, and J. Wöstemeyer, "Partial purification and characterisation of the peptide precursors of the cocoa-specific aroma components", Food Chem., Vol. 192, pp. 706713, 2016.

[17] K. Hori, T. Kiriyama, and K. Tsumura, "A Liquid Chromatography Time-of-Flight Mass Spectrometry-Based Metabolomics Approach for the Discrimination of Cocoa Beans from Different Growing Regions", Food Anal. Methods, Vol. 9, No. 3, pp. 738-743, 2016.

[18] D. Bertoldi, A. Barbero, F. Camin, A. Caligiani, and R. Larcher, "Multielemental fingerprinting and geographic traceability of Theobroma cacao beans and cocoa products", Food Control, Vol. 65, pp. 46-53, 2016.

[19] A. Marseglia, D. Acquotti, R. Consonni, L. R. Cagliani, G. Palla, and A. Caligiani, "HR MAS $1 \mathrm{H}$ NMR and chemometrics as useful tool to assess the geographical origin of cocoa beans Comparison with HR 1H NMR", Food Res. Int., Vol. 85, pp. 273-281, 2016.

[20] J. Van Durme, I. Ingels, and A. De Winne, 
"Inline roasting hyphenated with gas chromatography - mass spectrometry as an innovative approach for assessment of cocoa fermentation quality and aroma formation potential", Food Chem., Vol. 205, pp. 66-72, 2016.

[21] E. Allegrucci, S. E. Reichenbach, F. Magagna, C. Cordero, E. Liberto, A. Guglielmetti, C. Bicchi, and G. Gobino, "Comprehensive Chemical Fingerprinting of High-Quality Cocoa at Early Stages of Processing: Effectiveness of Combined Untargeted and Targeted Approaches for Classification and Discrimination", J. Agric. Food Chem., Vol. 65, No. 30, pp. 6329-6341, 2017.

[22] H. Rottiers, D. A. Tzompa Sosa, L. Van de Vyver, M. Hinneh, H. Everaert, J. De Wever, K. Messens, and K. Dewettinck, "Discrimination of Cocoa Liquors Based on Their Odor Fingerprint: a Fast GC Electronic Nose Suitability Study", Food Anal. Methods, Vol. 12, No. 2, pp. 475-488, 2019.

[23] M. E. Ali, M. Kashif, K. Uddin, U. Hashim, S. Mustafa, and Y. Bin Che Man, "Species Authentication Methods in Foods and Feeds: The Present, Past, and Future of Halal Forensics", Food Anal. Methods, Vol. 5, No. 5, pp. 935-955, 2012.

[24] K. Triyana, M. Taukhid Subekti, P. Aji, S. Nur Hidayat, and A. Rohman, "Development of Electronic Nose with Low-Cost Dynamic Headspace for Classifying Vegetable Oils and Animal Fats", Appl. Mech. Mater., Vol. 771, pp. 50-54, 2015.

[25] S. N. Hidayat and K. Triyana, "Optimized back-propagation combined with radial basic neural network for improving performance of the electronic nose: Case study on the fermentation process of tempeh", AIP Conf. Proc., Vol. 1755, 2016.

[26] S. N. Hidayat, T. R. Nuringtyas, and K. Triyana, "Electronic Nose Coupled with Chemometrics for Monitoring of Tempeh Fermentation Process", In: Proc. of 2018 4th Int. Conf. Sci. Technol., Vol. 1, pp. 1-6, 2018.

[27] S. N. Hidayat, K. Triyana, I. Fauzan, and T. Julian, "The Electronic Nose Coupled with Chemometric Tools for Discriminating the Quality of Black Tea Samples In Situ", Chemosensors, Vol. 7, No. 3, p. 29, 2019.

[28] V. O. S. Olunloyo, T. A. Ibidapo, and R. R. Dinrifo, "Neural network-based electronic nose for cocoa beans quality assessment", Agric. Eng. Int., Vol. 13, No. 4, pp. 1-17, 2011.

[29] J. Tan and W. L. Kerr, "Characterizing cocoa refining by electronic nose using a Kernel distribution model", LWT - Food Sci. Technol., Vol. 104, pp. 1-7, 2019.

[30] Badan Standardisasi Nasional, Biji kakao SNI 2323:208. Jakarta, Indonesia, 2008.

[31] J. Yan, X. Guo, S. Duan, P. Jia, L. Wang, C. Peng, and S. Zhang, "Electronic Nose Feature Extraction Methods: A Review", Sensors, Vol. 15, No. 11, pp. 27804-27831, 2015.

[32] F. Westad and F. Marini, "Validation of chemometric models - A tutorial", Anal. Chim. Acta, Vol. 893, pp. 14-24, 2015.

[33] A. Géron, Hands-On Machine Learning with Scikit-Learn and TensorFlow. United States of America: O'Reilly, 2017.

[34] J. Moolayil, Learn Keras for Deep Neural Networks. Berkeley, CA: Apress, 2019.

[35] A. Caligiani, L. Palla, D. Acquotti, A. Marseglia, and G. Palla, "Application of $1 \mathrm{H}$ NMR for the characterisation of cocoa beans of different geographical origins and fermentation levels", Food Chem., Vol. 157, pp. 94-99, 2014. 